\title{
Persistent Chaos in High Dimensions
}

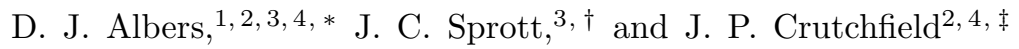 \\ ${ }^{1}$ Max Plank Institute for Mathematics in the Sciences, Leipzig 04103, Germany \\ ${ }^{2}$ Computational Science and Engineering Center and Physics Department, \\ University of California, Davis, One Shields Ave, Davis CA 95616 \\ ${ }^{3}$ Physics Department, University of Wisconsin, Madison, WI 53706 \\ ${ }^{4}$ Santa Fe Institute, 1399 Hyde Park Road, Santa Fe, NM 87501
}

(Dated: October 22, 2018)

\begin{abstract}
An extensive statistical survey of universal approximators shows that as the dimension of a typical dissipative dynamical system is increased, the number of positive Lyapunov exponents increases monotonically and the number of parameter windows with periodic behavior decreases. A subset of parameter space remains in which topological change induced by small parameter variation is very common. It turns out, however, that if the system's dimension is sufficiently high, this inevitable, and expected, topological change is never catastrophic, in the sense chaotic behavior is preserved. One concludes that deterministic chaos is persistent in high dimensions.
\end{abstract}

PACS numbers: 05.45.-a, 89.75.-k, 05.45.Tp, 89.70.+c, 89.20.Ff

Keywords: Chaos, high dimensions, dynamical systems, structural stability, Lyapunov exponents

Physical theory attempts to describe and predict the natural world by expressing observed behavior and the governing balance of forces formally in mathematical models - models that can only be approximate representations. Empirically, many natural phenomena persist even when control parameters and external conditions vary. For example, the essential character of fully developed fluid turbulence is little affected if one slightly changes the energy flux that drives it or if a small dent is made in the containing vessel's wall. In building a theory of a system exhibiting this kind of dynamical persistence, one hopes that, despite its approximations, one's model also has this persistence.

A century of analyzing nonlinear dynamical systems, however, has lead to an apparent inconsistency with this goal. Since the days of Poincaré's development of qualitative dynamics, mathematicians and physicists have probed differential equations to test their solutions for different kinds of stability. Poincaré's discovery of deterministic chaos 16] demonstrated that at the most detailed level, there was inherent instability of system solutions: change the initial condition only slightly and one finds a different state-space trajectory develops rapidly. Later studies showed that there was also an instability in behavior if the equations or parameters were changed only slightly 8, 15. Even arbitrarily small functional perturbations to the governing dynamic leads to radical changes in behavior - from unpredictable to predictable behavior, for example. The overall conclusion has been that nonlinear, chaotic systems are exquisitely sensitive, amplifying arbitrarily small variations in initial and boundary conditions and parameters to macroscopic scales.

\footnotetext{
*Electronic address: albers@cse.ucdavis.edu

${ }^{\dagger}$ Electronic address: sprott@physics.wisc.edu

${ }^{\ddagger}$ Electronic address: chaos@cse.ucdavis.edu
}

How can one reconcile this with the observed fact of dynamical persistence in many large-scale systems? We take dynamical persistence to mean that a behavior type - e.g., equilibrium, oscillation, chaos - does not change with functional perturbation or parameter variation. Here we describe the results of a Monte Carlo survey which empirically demonstrate that chaos is dynamically persistent if the dimension of a nonlinear system is sufficiently high. More constructively, we argue that a particular mechanism is responsible for persistent chaos.

Specifically, the survey shows that in large-scale systems dynamical sensitivity - when defined as breaking topological equivalences associated with structural stability [13], ergodicity [17], and statistical stability [3] - is typically benign and does not affect behavior types. Naturally, drastic changes in the invariant measure yield different observed dynamics, but the results indicate that this becomes increasingly less probable. Moreover, the instability associated with deterministic chaos dominates high-dimensional dynamical systems, except at extreme parameter settings.

Much of the intuition and motivation for our investigation comes from the analytical results found in abstract dynamical systems theory, but our construction and conclusions highlight a distinct difference. Said most simply, the number of dimensions of the dynamical system matters. That is, there is a qualitative difference between common behaviors in high- and low-dimensional dynamical systems. Beyond giving empirical evidence to support these conclusions, we introduce a definition of persistent chaos that suggests an alternative approach to the long-standing questions of dynamical stability and offer a mathematical conjecture on the mechanism underlying persistent chaos in high dimensions.

The spectrum of Lyapunov characteristic exponents (LCE) 7] will be our primary tool for analyzing and identifying behavior types since there is an equivalence 
between the number of negative and positive Lyapunov exponents and the number of global stable and unstable manifolds, respectively - structures that organize the state space and constrain behavior [18]. Therefore, in referring to topological variation here we mean a change in the number of positive Lyapunov exponents.

In order to give a complete representation of the space of all systems, we investigate typical behaviors in high dimensions using a class of dynamical systems that are known to be universal function approximators. (They are universal in that they approximate arbitrarily closely any $C^{r}$ mapping, cf. [10].) These are single-layer neural networks of the form

$$
x_{t}=\beta_{0}+\sum_{i=1}^{n} \beta_{i} \tanh s\left(\omega_{i 0}+\sum_{j=1}^{d} \omega_{i j} x_{t-j}\right),
$$

which are maps from $R^{d}$ to $R$. Here $n$ is the number of hidden units (neurons), $d$ the number of time lags which determines the system's input (embedding) dimension, and $s$ a scaling factor for the connection weights $w_{i j}$. The initial condition is $\left(x_{1}, x_{2}, \ldots, x_{d}\right)$ and the state at time $t$ is $\left(x_{t}, x_{t+1}, \ldots, x_{t+d-1}\right)$. The approximation theorems of Ref. 10] and time-series embedding results of Ref. 19. establish an equivalence between these neural networks and general dynamical systems; cf. [2].

In the Monte Carlo survey we sample the $(n(d+1)+1)$ dimensional parameter space taking (i) $\beta_{i} \in[0,1]$ uniformly distributed and rescaled to satisfy $\sum_{i=1}^{n} \beta_{i}^{2}=n$, (ii) $w_{i j}$ as normally distributed with zero mean and unit variance (which is adjusted with $s$ ), and (iii) the initial $x_{j} \in[-1,1]$ as uniform. These distributions - denoted $m_{\beta}, m_{w}(s)$, and $m_{I}$-form a product measure on the space of parameters and initial conditions - the survey's results then are statistical estimates with respect to this product measure. We use the $s$ parameter as the primary control as it gives the magnitude of the argument of $\tanh (x)$. When $x \approx 0$ Eq. (II) is linear and one finds fixed points and limit cycles; when $|x| \gg 1$ the output is binary and one finds $2^{n}$ different periodic states; and for $|x|$ between these extremes we find the nonlinear behavior we will focus on.

We define persistent chaos in terms of the LCE spectrum as follows:

Definition 1 (Degree- $p$ Persistent Chaos) Assume a discrete-time map $f$ that takes a compact set to itself. The map has persistent chaos of degree-p if there exists an open subset $U$ of parameter space, such that, for all $\xi \in U$ and a given open set $\mathcal{O}$ of initial conditions, $\left.f\right|_{\xi}$ retains $p \geq 1$ positive Lyapunov exponents.

Persistent chaos ( $p$-chaos) of degree $p$ is our notion of dynamical equivalence on an open set of parameter space. This differs from that of a robust chaotic attractor of Refs. [4] and [5], for example, in that we do not require the attractor to be unique on the subset $U$. This is an important distinction since, physically, there is little evidence indicating that such strict forms of uniqueness are present in many complex physical systems 12 and, technically, uniqueness is significantly more difficult

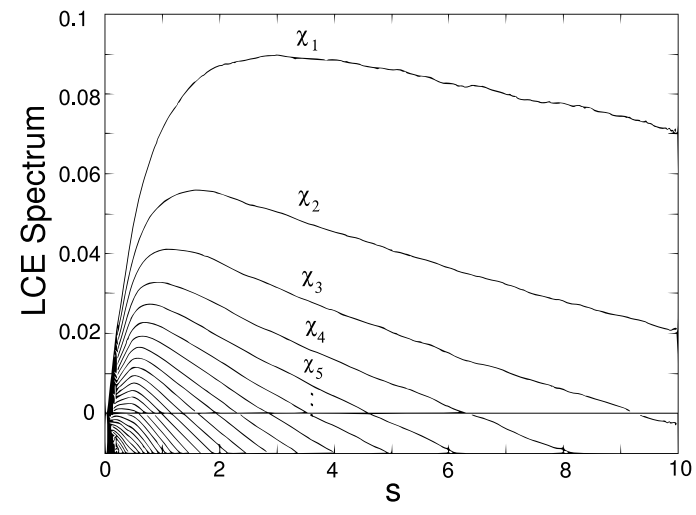

FIG. 1: LCE spectrum as a function of scale factor $s$ for a network of 32 neurons and 64 dimensions. (15000 total timesteps; 5000 initial time-steps to arrive on the attractor.)

to demonstrate. Indeed, alternative dynamical equivalence frameworks - such as nonuniform partial hyperbolicity [ 6$]$ — were invented to circumvent problems with nonuniqueness.

Figure [1] represents the typical scenario for the LCE spectra 21] of the high-dimensional neural networks as a function of $s$. Here typical refers to what was observed in $>99 \%$ of the 15800 networks with $n \geq 32$ and $d>32$ given $m_{\beta}, m_{w}(s)$, and $m_{I}$ as defined earlier. Important features to notice include the lack of periodic windows and that the LCEs vary continuously with $s$ and have a single maximum (up to numerical fluctuations). The survey also reveals that the maximum number of positive Lyapunov exponents is approximately $d / 4$ and the attractors' Kaplan-Yorke dimension is roughly $d / 2$ [1]. As $d$ increases, the length of the $s$-intervals between LCE zero-crossings decreases as $\sim d^{-1.92}$. These properties contrast sharply with the familiar low-dimensional scenarios where one typically encounters a preponderance of stable behavior and periodic windows and the LCEs vary in a discontinuous manner with control parameters. (A more complete analysis of these observations is found in Ref. 2].) Moreover, this scenario is different from low-entropy, spatially-extended systems discussed in [9] or 11. In our systems, the effective dimension is irreducibly high and the uniformity of the dynamics is more robust.

These observations complement those from a previous study of chaos in neural-network continuous-time differential equations [20]. There, a mean-field analysis, which assumes that inputs are statistically independent (and which does not apply in the present case), also suggested that chaos should be common in high dimensions.

In light of Fig. 1 and the fact that LCE zero crossings become asymptotically dense $\left(\left|U_{i}\right| \sim d^{-1.92}\right.$ for $i<20, d \leqq 128$ ) we propose the following dynamical mechanism for persistent chaos. For a finite but arbitrarily large number of dimensions along an $s$ intervale.g., $s \in(2,8)$ - there is an asymptotically dense, always countable sequence of parameter values that have an LCE 
transversally crossing through zero. Thus, a continuous path along an $s$-interval yields inevitable, but noncatastrophic (i.e. $p>1$ ) topological change. This implies that when varying parameters, periodic and quasiperiodic windows will not exist in chaotic regions of parameter space of dynamical systems with a sufficiently large number of positive exponents (i.e., when entropy rate is large). The lack of dense periodic and quasiperiodic windows is a necessary condition for $p$-chaos.

To test this picture, we analyzed the existence of periodic and quasiperiodic windows along $s \in(1,4)$ in networks with $n=32$ and $d$ ranging from 8 to 128 and with an ensemble of 700 networks per $n$ and $d$. We observed that (i) the mean fraction of networks with periodic and quasiperiodic windows decreases like $\sim d^{-1.3}$, (ii) the mean number of windows decreases like $\sim d^{-2}$, and (iii) the window lengths increase linearly with increasing $d$. These observations are insensitive to increments in $s$ as long as $\Delta s \leq 0.005$. As the dimension increased above 64 the only networks with periodic windows had windows that persisted for most of the $s$-interval under consideration. That is, as dimension was increased, periodic windows became increasingly rare. When they were observed, however, they were neither small nor intermittent, but instead dominated the dynamics.

To explore the full parameter space systematically, one can fix $s$ and vary the weights with random perturbations of a given size. We surveyed networks with parameters varied in a $(n(d+1)+1)$-ball with its center fixed at $s$ noting that the results are insensitive to $s$ variation in the chaotic portion of the $s$-interval; i.e., nearby $s$ values yield identical results. Similarly, the results are insensitive to perturbation size - weight variation on scales ranging from $10^{-10}$ to 1 yield similar results.

The results are shown in Fig. 2] the probability of observing periodic windows decreases as the dimension increases. Each data point corresponds to the probability of finding a system with a periodic orbit among a set of 700 networks at a given $n$ and $d$ and each perturbed 100 times. The range of weight perturbations was $10^{-3}$ with $s=3$. We found that the probability of periodic networks decreases as $d^{-2}$. Thus, as dimension increases the systems are far less likely to display periodic windows and, as a consequence, become more persistently chaotic.

While this is strong evidence for the disappearance of periodic windows in parameter space with increasing dimension, a stronger argument follows from our observation that the fraction of networks with windows decreases less quickly $\left(\sim d^{-1.3}\right)$ than the overall probability of windows $\left(\sim d^{-2}\right)$. Thus, periodic windows which do exist are concentrated in an ever-decreasing fraction of networks and those with one periodic window are more likely to have many periodic windows.

To quantify the degree of $p$-chaos for an ensemble of mappings with our construction consider the distribution of LCE's at a fixed $s$, noting that $p$ depends on $s$. If one takes $p$ to be the mean number of positive LCE's, then $p$ increases monotonically like $\sim d / 4[1]$ when $s$ is set to give the maximum number of positive LCEs. If one

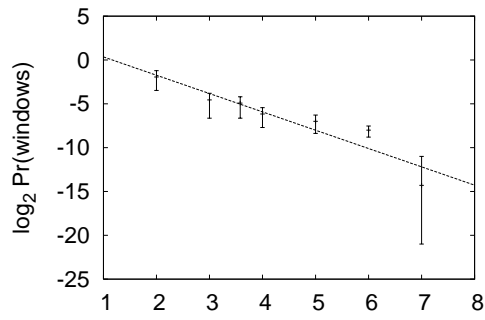

FIG. 2: Log probability of periogac behavior versus log dimension for 700 cases per $d$. Each case has all the weights perturbed on the order of $10^{-3} ; 100$ times per case. The best-fit line is $\sim 1 / d^{2}$.

considers $p$ to be the mean number of positive LCE's minus 3 standard deviations, a more conservative estimate, $p>0$ at $d=32$ and increases like $\sim 4 \log d$ at $s=3[1]$. We will refrain from arguing for a best definition of $p$ and simply note that $p$ increases with $d$ monotonically. Together with the periodic window data, this implies that while the parameter change required to alter the absolute number of positive exponents decreases, the perturbation required for all the positive exponents to vanish grows substantially. Thus, the chance of falling into a periodic window vanishes in proportion, and chaos becomes persistent over a considerable portion of parameter space.

These observations and detailed analysis of 400 fourdimensional dynamical systems and 200 64-dimensional dynamical systems, as well as many of intermediate dimension, leads to the following view of dynamic (topological) variation with parameter change. All of the LCEs that become positive are negative for very small and very large values of $s$ - the LCE spectra exhibit a single maximum. As the dimension $d$ is increased, their variations decrease and their $s$-dependence becomes smoother; recall Fig. 11 Moreover, with increasing dimension the number of positive exponents increases monotonically 1]. Finally, the distance between LCE zero-crossings, above the maximum, decreases with dimension as shown schematically in Fig. 3 .

Figure 3 is a graphical depiction of the hypothesized persistence mechanism - a plot of the $s$ axis transversally intersected by LCEs. In sufficiently high dimensions, the subsets $U_{i}$ shrink and eventually fall below any resolvability. The result, then, is twofold: one observes continuous topological change (bifurcations), but this is never catastrophic. One sees persistent chaos of varying degrees. The onset of sufficiently high dimension for this to occur for our dynamical systems was observed to be $d \geq 30$. These investigations lead to the following conjecture for persistent chaos in high dimensions:

Conjecture 1 Assume $f$ as in Eq. (1) and a sufficiently large number $d$ of dimensions and number $k=n(d+2)+1$ of parameters. There exists a large [2] Lebesgue measurable set of $s \in R^{1}$ with respect to $m_{\beta}, m_{w}(s)$, and $m_{I}$, for which chaos will be degree-p persistent. Moreover, $p \rightarrow \infty$ as $d \rightarrow \infty$.

Since our networks are universal function approximators, 


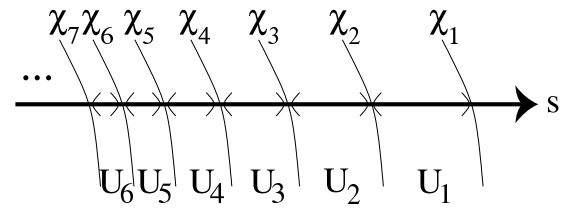

FIG. 3: Lyapunov spectrum versus network nonlinearity $s$ : $U_{i}$ 's are the open sets in parameter space where structural stability is believed to persist. The $\left|U_{i}\right|$ parameter intervals shrink like $\sim d^{-1.92}$ as the dimension increases.

this behavior should be observed in typical nonlinear high-dimensional dynamical systems.

Two comments are in order. First, the existence of chaos as a persistent behavior type depends on dimension. The subset of parameter space in which chaos becomes persistent increases in size (with respect to Lebesgue measure) as the dimension of the dynamical system increases. This is due both to the increase in the number of positive LCEs (given a sufficient increase in $n$ ) and to a decrease in the appearance of periodic windows. Second, persistence is related to the number of (linearly independent) parameters in the dynamical system. The number of neurons in the network effectively controls the entropy rate 14 - that is, increasing the number of neurons increases the entropy rate, number of positive exponents, and the maximum of the largest exponent. Increasing $n$ simply increases the degree $(p)$ of the persistent chaos, but the mechanism for persistent chaos remains, due to the decreasing probability of periodic windows. Networks with few parameters exhibit considerably less persistent chaos.

In this way high entropy-rate systems are more persistent with respect to functional and parameter perturbations. This is in accord with a wide range of experimental observations of such systems. Indeed, dynamical persistence is not a novel experience; often hydrodynamic engineers and plasma experimentalists expend much effort in attempts to eliminate persistent chaos. Here we described a mechanism in which the dynamical persistence of high-dimensional systems is retained under parameter perturbation, despite the fact that stricter notions of dynamical equivalence are violated. This sets the stage for more specific investigations of the statistical topology of stable and unstable manifolds in high-dimensional systems - investigations that, one hopes, will lead to predictive scaling theories for observed macroscopic properties that are grounded in microscopic dynamics.

We thank J. R. Albers, R. A. Bayliss, K. Burns, W. D. Dechert, D. Feldman, J. Robbin, C. R. Shalizi, and J. Supanich for helpful discussions. This work was partially supported at the Santa Fe Institute under the Networks Dynamics Program funded by the Intel Corporation and under the Computation, Dynamics, and Inference Program via SFI's core grants from the National Science and MacArthur Foundations. Direct support for DJA was provided by NSF grants DMR-9820816 and PHY9910217 and DARPA Agreement F30602-00-2-0583.
[1] D. J. Albers. A qualitative numerical study of highdimensional dynamical systems. $\mathrm{PhD}$ thesis, University of Wisconsin in Madison, 2004.

[2] D. J. Albers and J. C. Sprott. Structural stability and hyperbolicity violation in large dynamical systems. Submitted, http://arxiv.org/pdf/nlin.CD/0408011.

[3] J. F. Alves and M. Viana. Statistical stability for robust classes of maps with non-uniform expansion. Ergod. Th. and Dynam. Sys., 22:1-32, 2002.

[4] M. Andrecut and M. K. Ali. Robust chaos in smooth unimodal maps. Phys. Rev. E, 64:025203, 2001.

[5] S. Banerjee, J. A. Yorke, and C. Grebogi. Robust chaos. Phys. Rev. Lett., 80:3049-3052, 1998.

[6] L. Barreira and Ya. Pesin. Lyapunov exponents and smooth ergodic theory. AMS, 2002.

[7] G. Benettin, L. Galgani, A. Giorgilli, and J.-M. Strelcyn. Lyapunov characteristic exponents from smooth dynamical systems and for Hamiltonian systems; A method for computing all of them. Part 2: Numerical application. Meccanica, 15:21-30, 1979.

[8] J. D. Farmer. Phys. Rev. Lett., 55(4):351-355, 1985.

[9] P. Grassberger. Information content and perdictability of lumped and distributed systems. Physica Scripta, 40:346-353, 1989.

[10] K. Hornik, M. Stinchocombe, and H. White. Universal approximation of an unknown mapping and its derivatives using multilayer feedforward networks. Neural Networks, 3:551, 1990.

[11] K. Kaneko. Collapse of tori and genesis of chaos in dis- sipative systems. World Scientific, 1986.

[12] K. Kaneko. Dominance of Milnor attractors in globally coupled dynamical systems with more than $7 \pm 2$ degrees of freedom. Phys. Rev. E, 66:055201, 2002.

[13] R. Mañé. A proof of the $C^{1}$ stability conjecture. Publ. Math. IHES, 66:161-210, 1988.

[14] E. F. Manffra. Properties of systems with time-delayed feedback. PhD thesis, Univesitat Gesamthochschule Wuppertal, 2002.

[15] S. Newhouse. The abundance of wild hyperbolic sets and nonsmooth stable set for diffeomorphisms. Publ. Math. IHES, 50:101-151, 1979.

[16] H. Poincaré. Sur les propriétés des fonctions définies par les éuations aux différences partieles. Oeuvres. GauthirVillars, Paris, 1929.

[17] C. Pugh and M. Shub. Stable ergodicity. Bull. Amer. Math. Soc., 41:1-41, 2003.

[18] D. Ruelle. Characteristic exponents and invariant manifolds in Hilbert space. Ann. Math., 115:243-290, 1982.

[19] T. Sauer, J. Yorke, and M. Casdagli. Embedology. J. Stat. Phys., 65:579-616, 1991.

[20] H. Sompolinsky, A. Crisanti, and H. J. Sommers. Chaos in random neural networks. Phys. Rev. Lett., 1988.

[21] For a $d$-dimensional system the spectrum consists of $d$ LCEs: $\chi_{1} \geq \chi_{2} \geq \ldots \geq \chi_{d}$, where indexing gives a monotonic ordering.

[22] "Large" depends on $d$ : for $d=64$ we estimate a Lebesgue measure $m\left(I_{s}\right)<20$; higher $d$ remains an open question. 